\title{
Dalla statistica alla creazione di pittogrammi: un esempio di itinerario didattico contestuale nella scuola dell'infanzia
}

\section{From statistics to pictogram creation: an example of contextual educational path in pre-primary school}

\author{
Leyla Bernasconi ${ }^{\bullet}$, Alberto Piatti ${ }^{\circ}$ e Mario Bottinelli Montandon ${ }^{\circ}$ \\ - Scuola dell'infanzia di Bioggio - Svizzera \\ - Dipartimento formazione e apprendimento, SUPSI - Locarno, Svizzera \\ 凶leyla.bernasconi@edu.ti.ch, alberto.piatti@supsi.ch, mario.bottinelli@supsi.ch
}

Sunto / Questo itinerario didattico interdisciplinare (matematica ed educazione visiva) è stato realizzato in una scuola dell'infanzia del Canton Ticino seguendo un approccio contestuale. In una prima fase, i bambini hanno realizzato un intero processo statistico, dalla domanda di ricerca alla rappresentazione dei risultati. Sono state proposte due indagini: la prima volta a capire se nelle case degli allievi fossero presenti più gatti o più cani, la seconda per determinare se nella scuola dell'infanzia di Bioggio fossero presenti più allievi o più allieve. Questa prima fase ha messo in evidenza come la difficoltà principale dei bambini fosse la rappresentazione dei risultati, e in particolare la rappresentazione di classi di equivalenza (gatto/cane, maschio/femmina). Questo genere di difficoltà è stato quindi affrontato didatticamente con il linguaggio visivo del pittogramma, che ha accompagnato gli allievi attraverso diversi compiti di realtà. Lungo le ultime fasi del suo sviluppo, l'itinerario è stato strutturato per consolidare la capacità degli allievi di creare pittogrammi.

Parole chiave: scuola dell'infanzia; statistica; pittogrammi; approccio contestuale.
Abstract / This interdisciplinary learning itinerary (mathematics and visual education) was carried out in a kindergarten in Canton Ticino following a contextual approach. In a first phase, the boys and girls carried out a whole statistical process, from the research question to the representation of the results. Two surveys were proposed: the first one aimed at understanding if in the houses of the pupils there were more cats or more dogs, the second one aimed at determining if in the kindergarten of Bioggio (Canton Ticino) there were more pupils or more students. This first phase showed that the main difficulty of the boys and girls was the representation of the results, and in particular the representation of equivalence classes (cat/dog, male/female). This kind of difficulty was addressed instructionally with the visual language of the pictogram, which accompanied the pupils through various reality tasks. Along the last stages of its development, the itinerary was structured to consolidate the pupils' ability to create pictograms.

Keywords: pre-primary school; statistics; pictograms; contextual approach.

(C) 2021 Bernasconi Leyla, Piatti Alberto e Bottinelli Montandon Mario. Questo è un articolo Open Access, sottoposto a un processo di revisione tra pari a doppio cieco, pubblicato dal Centro competenze didattica della matematica e dal Servizio risorse didattiche e scientifiche, eventi e comunicazione del DFA-SUPSI in collaborazione con il DECS. L'articolo è distribuito sotto i termini della Licenza Creative Commons Attribuzione 4.0 Internazionale (CC BY 4.0) che permette di usare, condividere e modificare l'articolo su qualsiasi mezzo a patto che l'autore e la fonte originale siano citati. 


\section{Introduzione}

L'itinerario presentato in questo articolo è stato concepito nell'ambito di un corso di formazione continua sul tema della progettazione di itinerari didattici in matematica a partire da contesti reali, proposto dal Dipartimento formazione e apprendimento nel corso dell'anno scolastico 2019-2020. L'itinerario è stato realizzato in una sezione di scuola dell'infanzia di Bioggio (Canton Ticino, Svizzera) nello stesso anno scolastico.

\subsection{Inquadramento teorico dell'approccio contestuale}

Il percorso presentato si basa sull'assunto che progettare un itinerario didattico efficace è come progettare un'esperienza di vita, da realizzare insieme ai propri allievi e alle proprie allieve, che risulti coinvolgente per tutti e che produca come risultato un apprendimento. Metaforicamente, progettare un itinerario didattico è come concepire un viaggio da compiere insieme ai propri allievi e alle proprie allieve in un territorio inesplorato. Per poter raggiungere questo obiettivo, è però necessario che l'itinerario didattico sia immerso in un contesto di senso. Per definire il concetto di contesto adottiamo la definizione di Klassen:

«[...] propongo di definire il contesto come tutte le entità che sono correlate o che circondano un determinato elemento focale e che contribuiscono alla sensatezza di tutto l'insieme. L'elemento focale nella definizione può essere una conoscenza scientifica o un'abilità, un concetto o una competenza. La sensatezza di tale entità nodale emerge da fattori quali la familiarità, l'interazione sociale, l'attività, la riflessione, la relazione logica, la risposta emotiva ecc., che, nel senso della definizione, costituiscono il contesto per il concetto o la competenza».

(Klassen, 2006, p. 35, traduzione degli autori)

Il contesto permette all'allievo o all'allieva di dare senso, di contestualizzare, un concetto o una competenza, intrecciandolo con altre conoscenze, competenze ed esperienze di vita, ancorandolo così a una memoria a lungo termine. Come definizione operativa, un approccio contestuale è tale se favorisce, come indicato dal Piano di studio della scuola dell'obbligo ticinese (Dipartimento dell'educazione, della cultura e dello sport [DECS], 2015), «una visione sistemica del progetto educativo» (p. 55), ovvero quando accoglie quella dimensione della complessità in cui saperi, competenze ed esperienze sono tessuti insieme.

Klassen (2006) distingue cinque ambiti di contesto:

1. Il contesto pratico: che tipi di attività vengono svolte dagli allievi e dalle allieve? Con quali finalità e quali metodologie? In che luogo? Questo ambito di contesto è fondamentale per la motivazione e il coinvolgimento degli allievi e delle allieve. In questo senso è fondamentale che le attività proposte, nel caso delle situazioni-problema, siano autentiche, ovvero rappresentino dei problemi significativi nel contesto reale o narrativo di riferimento. Riprendendo la metafora del viaggio, questo ambito corrisponderebbe al tipo di attività svolta durante il viaggio: scoperta, socializzazione, festa, rilassamento ecc.

2. II contesto teorico: qualsiasi contesto si basa su conoscenze e teorie. Quali sono i concetti principali che sottostanno al contesto prescelto? Spesso le risorse cognitive mirate sono basate su questi concetti di fondo. Per questo ambito di contesto sono fondamentali la correttezza e la ricchezza dei concetti di fondo mobilitati. È dunque sempre utile scegliere contesti (reali o narrativi) di cui i//la docente abbia una conoscenza approfondita, o ancora meglio una passione. Nella metafora del viaggio, questo ambito di contesto corrisponderebbe alla destinazione o alle destinazioni del viaggio: dove vado? E a vedere cosa? 
3. Il contesto sociale: andare in vacanza da solo, o con il compagno o la compagna, o con la famiglia, o con un gruppo di persone sconosciute non è la stessa cosa. I compagni di viaggio influenzano in maniera preponderante l'esperienza vissuta. Lo stesso vale per un itinerario didattico: con chi svolgerò il percorso e con quali modalità?

4. Il contesto storico: le conoscenze e le teorie trattate a scuola spesso differiscono in modo importante dallo stato della ricerca e delle conoscenze accademiche sugli stessi ambiti. Spesso la trasposizione didattica, soprattutto in matematica, porta a presentare i concetti come se fossero puri, universali e immutabili. In questo senso, giova piuttosto presentare e impostare i concetti in tutta la loro umanità e variabilità. Le teorie sono di regola frutto di spiegazioni alternative e contrastanti, di dibattiti e conflitti, di esperimenti riusciti e falliti, di casualità, di problemi che si sono posti nel tempo ecc. Prendere in considerazione la storia dei diversi concetti e il loro sviluppo nel tempo consente di rendere viva la teoria e quindi di aumentare la motivazione e il coinvolgimento degli allievi e delle allieve. Chi di noi, visitando una grande città o una regione, ha trascurato completamente la sua storia? È possibile apprezzare veramente un luogo senza sapere nulla della sua storia e dello sviluppo che ha avuto?

5. Il contesto affettivo e narrativo: non c'è apprendimento senza emozioni ed esperienza in prima persona. Quali vissuti e quali emozioni I'allievo ricollega al contesto considerato? Quali esperienze ha già raccolto in questo contesto? Si tratta di un contesto narrativo oppure di un contesto reale legato alla quotidianità dell'allievo o dell'allieva?

\title{
1.1.1 Significato e senso di un concetto matematico
}

In quest'ottica, in didattica della matematica si distingue tra significato e senso di un concetto (Radford et al., 2011).

\begin{abstract}
«ll significato di un concetto è un costrutto storico, culturale e politico, condiviso all'interno di una comunità, e appartenente a una coscienza collettiva. La definizione di un concetto matematico, condiviso all' interno della comunità scientifica, può essere un esempio di significato di un concetto. Il significato di un concetto, pur essendo oggettivo, non è però, come si potrebbe facilmente pensare, una realtà statica e stabile, ma piuttosto il risultato di una serie di tensioni e visioni contrapposte all'interno della comunità di riferimento e come tale è in continua evoluzione».
\end{abstract}

(Piatti, 2015, p. 104)

In generale però,

«[...] quando si chiede a una persona di evocare un concetto matematico, spesso ciò che sorge spontaneo alla mente è legato a una o più esperienze o attività, più o meno concrete, che la persona ha vissuto e in cui quel concetto era coinvolto. L'insieme di tutte le esperienze che una persona ha vissuto con un dato concetto; le immagini, le parole, i ricordi, i gesti legati a quelle esperienze formano quello che gli autori definiscono il senso del concetto matematico. In altre parole, il senso sta alla coscienza individuale, come il significato sta alla coscienza collettiva, ma le coscienze individuali non sono e non potranno mai essere riproduzioni in scala della coscienza collettiva».

(Piatti, 2015, p. 104)

Il contesto affettivo e narrativo è l'ambito del contesto che maggiormente è influenzato dalle esperienze precedenti e che più differenzia le esperienze dei singoli allievi. Se pensiamo alla metafora del viaggio, spesso le destinazioni cui siamo maggiormente legati o per noi più significative sono quelle a cui possiamo collegare delle emozioni intense. Seguire un approccio contestuale significa prendere 
in considerazione tutti questi ambiti di contesto nella progettazione di percorsi che si traducano per gli allievi e le allieve in vere esperienze di vita, di viaggio.

\subsection{Inquadramento teorico del pittogramma}

La proposta del pittogramma segue l'impostazione pedagogico-didattica finora delineata, coinvolgendo aspetti che si riferiscono allo studio del disegno infantile e alla semiotica cognitiva (contesto teorico). Darras (1998) ha inquadrato il termine pittogramma nel dominio degli schemi: «l pittogrammi sono schemi convalidati da una comunità di utenti, il che ha permesso loro di stabilizzarsi nel tempo e, talvolta, anche di evolversi verso sistemi di scrittura» (Darras, 1998, p. 93, traduzione degli autori). Secondo il semiologo francese, inoltre, il pittogramma assolve un bisogno figurativo più che visivo, nel senso che, disegnando, il bambino si pone sul piano della comunicazione visiva convenzionale (iconotipo). Considerare i tracciati infantili, al contrario, solo come fenomeni dell'espressione personale, rifacendosi a categorie di giudizio estetico derivate dall'arte, equivarrebbe a trascurare degli schemi grafici il fatto che «ogni iconotipo è un potenziale pittogramma e le produzioni dei bambini ne sono i laboratori» (Darras, 1998, p. 93, traduzione degli autori).

Il focus sull'espressione grafica come comunicazione consentirebbe dunque un'interpretazione «semiotico-pragmatica» (Rickenmann, 2001) degli schemi prodotti dai bambini, spostando l'attenzione del docente dal prodotto al processo, o meglio dall'immagine al segno, cioè quell'articolazione figurativa che «indica il tipo di lavoro semiotico che il ricevente deve fare per ricostruire il suo significato usando regole comuni del sistema» (Rickenmann, p. 238, traduzione degli autori). La rilevanza sociale e «consensuale» del pittogramma infantile (Rickenmann, 2001, p. 235) si ritrova anche nella segnaletica contemporanea. A questo proposito, Massironi (1982) nota che ai segnali è attribuito il compito di «trasmettere delle informazioni essenziali a un grande numero di persone di lingua diversa, ma che hanno tratti socio-culturali comuni e a cui non è stato fornito alcun addestramento per affrontare la decodifica di tali messaggi» (p. 104). Ancora secondo Darras, la segnaletica come strategia di comunicazione visiva ha lo scopo primario di economizzare le risorse cognitive di chi osserva. Anche quando la loro elaborazione avviene empiricamente, i pittogrammi della segnaletica manifestano sempre la preoccupazione di essere percepiti in quanto tali, «facilitando il riconoscimento, le inferenze e la memorizzazione» (Darras, 2005, p. 4, traduzione degli autori). Grazie alla loro qualità di facilitatori, pertanto, i pittogrammi «anticipano il lavoro cerebrale riducendo, comprimendo, separando, categorizzando, neutralizzando e stereotipando le informazioni» (Darras, 2005, p. 4, traduzione degli autori). Per Massironi (1982), inoltre, è essenziale che i pittogrammi consentano all'osservatore «la maggior velocità di lettura possibile» (p. 107).

Il pittogramma come segno in un contesto si distingue per alcune caratteristiche ricorrenti: occupa un'area spaziale riservata, per esempio all'interno di un riquadro o cartello più o meno standardizzato; adotta una grafica lineare, a volte potenziata con informazioni testuali, e colori convenzionali a tinte piatte. Darras nota che «tutte queste caratteristiche ci permettono di aumentare il divario con altri potenziali segni dell'ambiente e di massimizzare la loro distinzione e individuazione» (Darras, 2005, p. 4, traduzione degli autori).

Per tornare brevemente all'impronta comunicativa del pittogramma come segno in contrapposizione al suo darsi come immagine, è interessante riportare la posizione di Massironi (1982, p. 104), per il quale esso costituisce una zona di confine fra il linguaggio, che procede per concetti, e la percezione, che procede invece per oggetti. In effetti, «i simboli grafici, informativi, si pongono in uno spazio compreso fra la definizione verbale di un concetto, generalmente non complesso, e la rappresentazione illustrativa, depurata di tutti gli attributi di singolarità» (Massironi, 1982, p. 111). Ma l'indicazione di Massironi è da leggere, per quanto ci concerne, soprattutto in chiave pedagogico-didattica, nel senso che permette di illuminare quella zona del lavoro formativo in cui le dimensioni disciplinari devono necessariamente incontrarsi: immagine e parola (oggetto percepito e concetto), infatti, sono 
codici della comunicazione che nel pittogramma trovano un'occasione di dialogo costruttivo. Del resto, è ben conosciuta, grazie all'opera e al pensiero del pedagogista Loris Malaguzzi (1920-1994), la capacità del bambino in età prescolare di mobilitare simultaneamente $i$ «100 linguaggi». Si impone di base la necessità di comunicare, che il bambino evade adoperando di volta in volta il linguaggio che reputa più efficace. Ma è una strategia comune anche agli adulti, come nota Massironi:

«[...] quando un sistema codificato in un certo modo (ad esempio il linguaggio) non ce la fa più, ne subentra un altro codificato diversamente [...] a fornire quel tanto d'informazione in più che il primo sistema non poteva dare [...]. Dove la scrittura trova degli ostacoli all'esaustività, subentra il disegno o l'illustrazione».

(Massironi, 1982, p. 111)

Alla scuola dell'infanzia è quindi utile, oltre che in sintonia con i processi di significazione adottati spontaneamente dai bambini, proporre situazioni in cui la codifica semantica segue percorsi cognitivi reversibili, integrando e avvicendando numero, linguaggio discorsivo e figurazione:

«[...] tra i vari percorsi della significazione vi è una continuità e il passaggio dall'uno all'altro è estremamente sfumato e non così netto come le varie gerarchie preposte fra i sistemi di segni, o gli esami condotti su universi separati potrebbero far pensare».

(Massironi, 1982, p. 112)

\section{Struttura dell'itinerario}

L'itinerario si presenta come una sequenza di tre fasi. La particolarità principale risiede nel fatto che le tre fasi non sono state concepite a priori nel loro insieme, ma ognuna è stata creata a seguito di un bisogno emerso lungo il percorso. Globalmente l'itinerario è stato rivolto principalmente ai venti bambini del secondo anno obbligatorio, i quali hanno lavorato al pomeriggio, nel momento a loro dedicato, per due pomeriggi a settimana nel corso di cinque mesi, per un totale di circa 40 ore lezione. Si è lavorato in grande gruppo, per il lancio degli stimoli e le riflessioni a conclusione delle varie fasi, in piccoli gruppi o a coppie per le sperimentazioni e lo svolgimento dei lavori.

In questo paragrafo presentiamo le principali scelte strutturali e didattiche effettuate durante il percorso. Nel par. 3 , invece, descriveremo più in dettaglio quanto avvenuto nella sperimentazione.

\subsection{Prima fase: la statistica}

\subsubsection{Indagine sugli animali domestici: ci sono più gatti o cani?}

A seguito del lancio dello stimolo "Secondo voi, nelle vostre case ci sono più gatti o più cani?» $i$ bambini hanno raccolto e rappresentato le informazioni necessarie per rispondere alla domanda, in un primo momento in modo spontaneo. È seguito un momento di istituzionalizzazione dei dati, attraverso la creazione di un cartellone riassuntivo.

\subsubsection{Indagine sul genere: ci sono più maschi o femmine?}

Inizialmente la domanda «Nella nostra scuola, ci sono più maschi o più femmine?» è stata ristretta ai venti bambini del gruppo dei bambini del secondo anno obbligatorio. In seguito, ha fatto invece riferimento a tutta la sede. II bisogno emergente è stato quello di stilizzare la forma grafica e questo ha 
portato a un lavoro sulla figura umana. I dati relativi al numero di maschi e femmine sono stati raccolti su un foglio. L'attività era volta a sviluppare le seguenti competenze, previste dal Piano di studio della scuola dell'obbligo ticinese (DECS, 2015): (i) attuare una serie di tentativi volti ad affrontare e risolvere una data situazione numerica derivante da un contesto famigliare (Esplorare e provare); (ii) rappresentare situazioni numeriche espresse in forma linguistica con parole, disegni, schemi, frecce, istogrammi (Matematizzare e modellizzare) e (iii) descrivere e presentare le proprie scelte prese per affrontare una situazione numerica in modo tale che risultino comprensibili per gli altri (Comunicare e argomentare). Inoltre, I'attività permette di sviluppare le competenze di collaborazione (Competenze trasversali) e di approfondire il contesto di formazione generale del Vivere assieme ed educazione alla cittadinanza.

In questa prima fase, sono stati scelti due contesti molti vicini alla quotidianità dei bambini e delle bambine: i propri animali domestici e i propri compagni e le proprie compagne (contesto affettivo e narrativo). I problemi posti, in tutta la loro semplicità, hanno rappresentato delle vere situazioni problema, che hanno richiesto di sviluppare complesse (per i bambini e le bambine) strategie di conteggio e rappresentazione (contesto teorico e pratico) in un ambito ben conosciuto. A livello sociale, l'attività è stata strutturata in modo articolato, per passare da una prima riflessione individuale a un'attività di classe, privilegiando in particolare la collaborazione tra allievi/e (contesto sociale). In questa fase, così come nelle seguenti, non è stato considerato esplicitamente l'ambito storico.

L'indagine su maschi e femmine ha messo in evidenza la necessità per i bambini e le bambine di sviluppare la capacità di rappresentare in maniera schematica delle entità del mondo reale. Durante la trascrizione sul foglio, i bambini si sono resi conto della difficoltà e del tempo necessario per disegnare ogni bambino presente, vista la tendenza a voler arricchire di dettagli tutti i protagonisti del disegno. Da una discussione è emerso il bisogno di trovare una strategia che risultasse più sostenibile in termini di tempo ed energie, più funzionale e visibile a colpo d'occhio. Si è deciso quindi di proporre un ciclo di attività sul tema dei pittogrammi. Si è proposta una prima serie di situazioni legate ai pittogrammi delle ferrovie federali svizzere, che sono stati interpretati e ricreati. Una seconda serie ha visto invece i bambini e le bambine concentrarsi sulla creazione di pittogrammi che servissero a identificare i diversi spazi del nuovo edificio scolastico di imminente inaugurazione. Entrambe le attività erano volte a sviluppare le seguenti competenze, previste dal Piano di studio della scuola dell'obbligo ticinese (DECS, 2015): (i) procedere per prove e tentativi nella manipolazione e osservazione di figure assegnate o di motivi corrispondenti (Esplorare e provare); (ii) tradurre situazioni geometriche - che coinvolgono figure o simmetrie - in rappresentazioni figurali (disegni, schemi, percorsi con frecce ecc.) o a parole (Matematizzare e modellizzare) e (iii) orientarsi e orientare persone o oggetti nello spazio reale usando termini specifici (Eseguire e applicare). Inoltre, I'attività permetteva di sviluppare competenze di comunicazione (Competenze trasversali) e di continuare ad approfondire il contesto di formazione generale del Vivere assieme ed educazione alla cittadinanza.

\subsection{Seconda fase: alla scoperta dei pittogrammi}

In questa fase sono stati scelti due contesti più ampi e in parte meno conosciuti dagli allievi e dalle allieve: i pittogrammi delle ferrovie svizzere (contesto storico) e la nuova sede scolastica (contesto affettivo e narrativo). Dal punto di vista disciplinare, ci si è spostati da un ambito numerico/statistico a uno prevalentemente geometrico, mentre gli aspetti relativi all'educazione visiva hanno preso una certa prevalenza nella didattica (contesto teorico). Anche in questo caso, le attività di riproduzione e creazione di pittogrammi hanno rappresentato delle situazioni problema autentiche (contesto pratico) ed è stata privilegiata una modalità collaborativa (contesto sociale).

Questa attività ha messo in evidenza la necessità per i bambini e le bambine di sviluppare le loro capacità di creare delle rappresentazioni della realtà utilizzando un insieme limitato di semplici figure. La ragione della scelta di permettere unicamente l'uso delle forme geometriche era facilitare la semplificazione e schematizzazione del disegno. 


\subsection{Terza fase: creiamo disegni con le sagome}

Nell'ultima fase è stato proposto un percorso didattico interdisciplinare tra matematica ed educazione visiva, dove i bambini e le bambine hanno creato delle figure utilizzando formine create appositamente. Dopo aver lavorato per un certo periodo con delle forme geometriche "piene" è emerso il bisogno di passare a forme che non fossero forzatamente convenzionali e a motivi più "leggeri" e adattabili. Sono state dunque introdotte delle sagome, o mascherine, che hanno permesso più libertà nel disegno. Dal punto di vista matematico, l'attività era volta sviluppare le seguenti competenze: (i) procedere per prove e tentativi nella manipolazione e osservazione di figure assegnate o di motivi corrispondenti (Esplorare e provare) e (ii) orientarsi e orientare persone o oggetti nello spazio reale usando termini specifici (Eseguire e applicare); dal punto di vista dell'educazione visiva, le competenze perseguite erano le seguenti, previste dal Piano di studio della scuola dell'obbligo ticinese (DECS, 2015): (i) identificare le nozioni spaziali elementari per analizzare e descrivere le componenti di un'immagine o di un'opera plastica (Percezione/Interpretazione) e (ii) inventare e produrre delle immagini, liberamente $\mathrm{o}$ in risposta a una consegna (Espressione e rappresentazione). Inoltre, I'attività permetteva di sviluppare le strategie di apprendimento (Competenze trasversali) e di approfondire il contesto di formazione generale delle Scelte e progetti personali.

In quest'ultima fase, si è deciso di riallacciarsi al contesto di riferimento dell'anno della sezione, ovvero gli uccelli (contesto affettivo e narrativo). Per quanto attiene l'ambito teorico, sono state introdotte nuove figure non standard e gli aspetti relativi all'educazione visiva hanno preso ancora maggior prevalenza (contesto teorico). II contesto pratico e il contesto sociale sono rimasti sostanzialmente invariati rispetto alla seconda fase.

Di seguito, illustriamo in dettaglio quanto avvenuto durante le tre fasi.

\section{Le fasi in dettaglio}

\subsection{Prima fase: la statistica}

\subsubsection{Indagine sugli animali domestici: ci sono più gatti o più cani?}

La prima fase dell'itinerario ha visto impegnati i venti bambini dell'ultimo anno di scuola dell'infanzia nel momento del pomeriggio, dalla metà di settembre alla prima settimana di ottobre 2019.

II tema è stato introdotto per mezzo di due albi illustrati di Simona Meisser (2010a, 2010b) Perché i cani odiano i gatti e Perché $i$ gatti odiano $i$ cani, con conseguente discussione su questi animali domestici.

Successivamente è stata proposta la domanda stimolo: "Secondo voi nelle nostre case ci sono più gatti o più cani?». Sono seguite risposte libere ispirate perlopiù dalla propria esperienza e dal proprio vissuto. Dopo un primo momento di sperimentazione in cui i bambini hanno rappresentato graficamente su un foglio il proprio animale domestico (a volte riportandone la cifra indo-araba, a volte riportando diversi animali sul foglio, altre volte indicando la quantità con dei trattini) è nata l'esigenza di elaborare una strategia comune e condivisa per effettuare una raccolta precisa dei dati. 


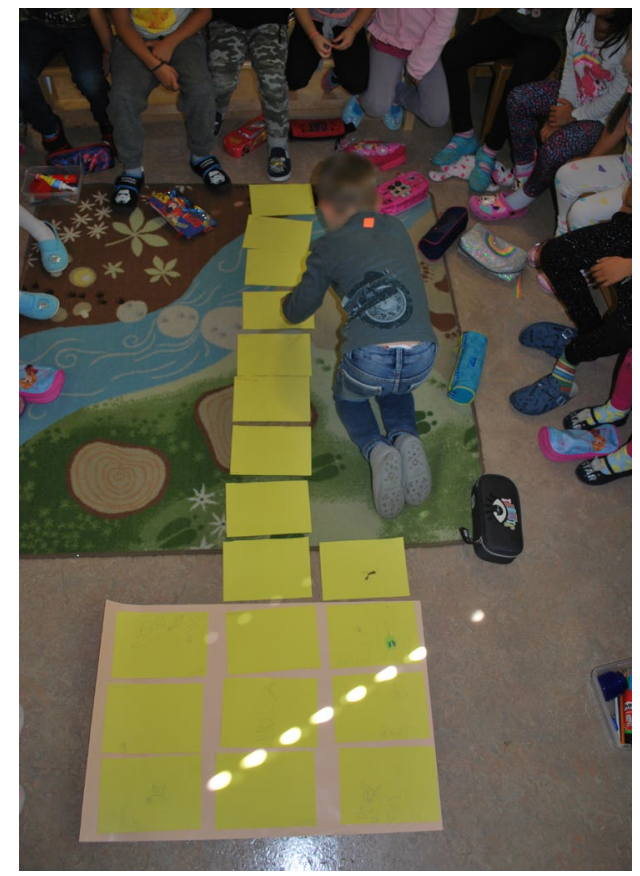

Figura 1. Un bambino conta i fogli con i disegni degli animali domestici.

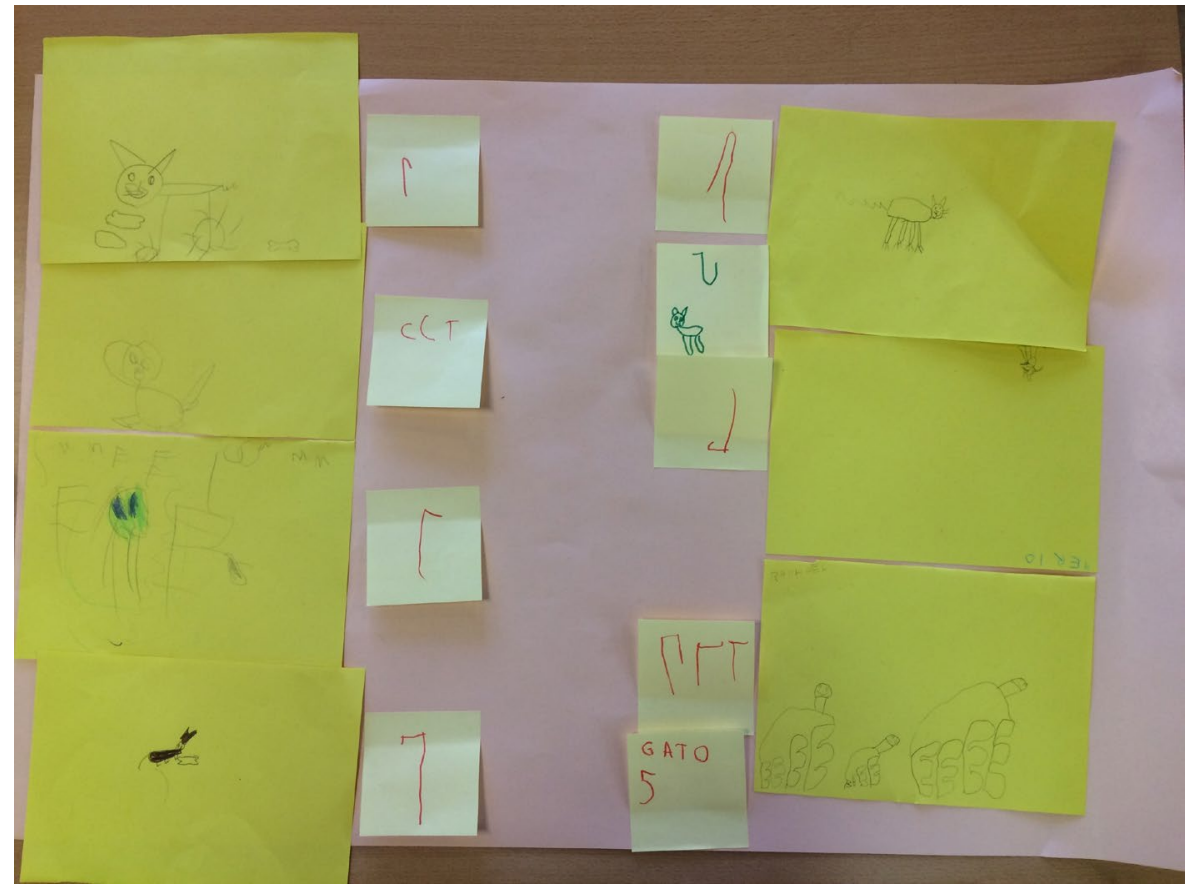

Figura 2. Tentativo di sintesi dei dati.

Una delle difficoltà riguardava coloro che non possedevano animali domestici: in alcuni casi venivano rappresentati e barrati, in altri casi il foglio veniva lasciato in bianco. Un'altra difficoltà è stata il conteggio: i bambini tendevano a contare i fogli o disporli in fila per valutare quale fosse la fila più lunga (Figura 1), senza tenere presente che non in tutti i casi un foglio contava come un'unità. I bambini hanno dunque proposto che, oltre al disegno, occorresse scrivere la cifra indo-araba, di solito più volte il numero 1, da conteggiare poi in sede di controllo finale (Figura 2).

Come illustrato nelle Figure 3 e 4, I'allestimento del cartellone ha permesso di sintetizzare i dati. 


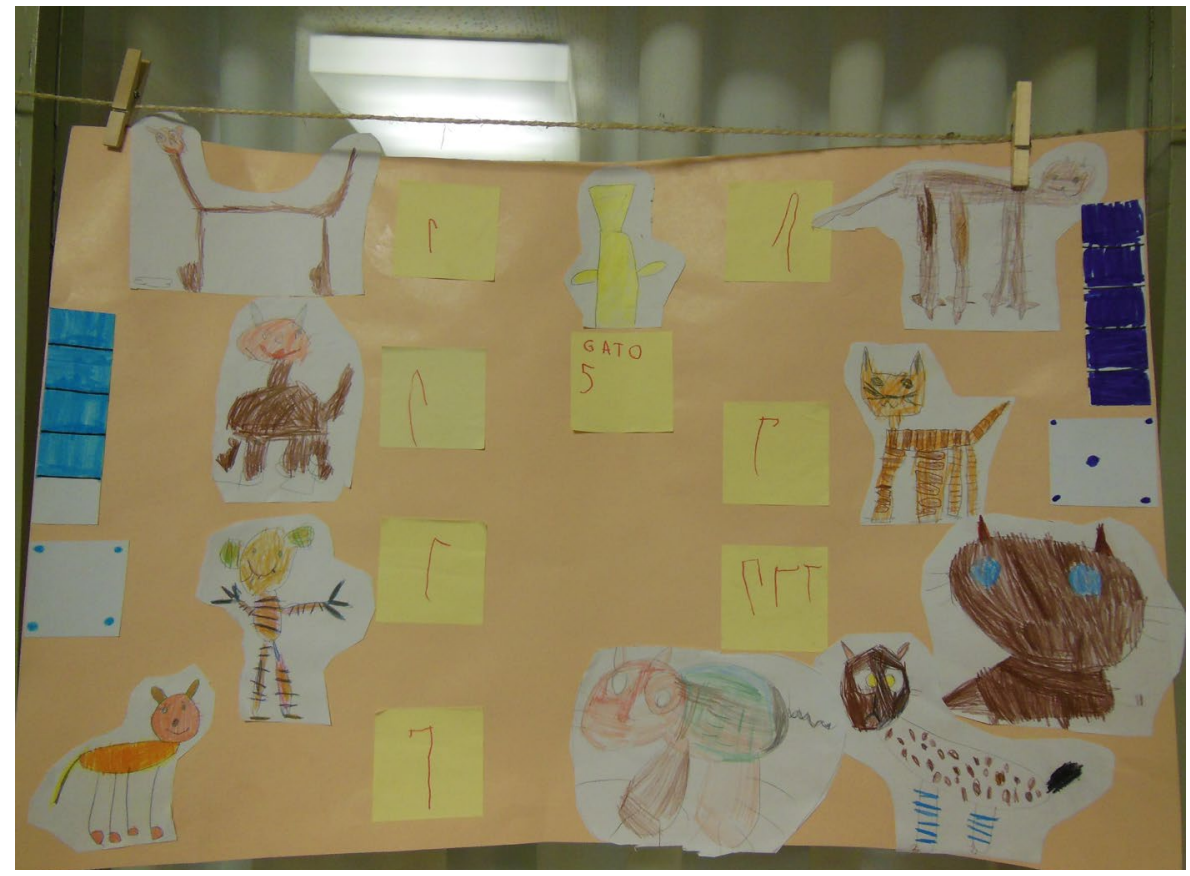

Figura 3. Cartellone riassuntivo

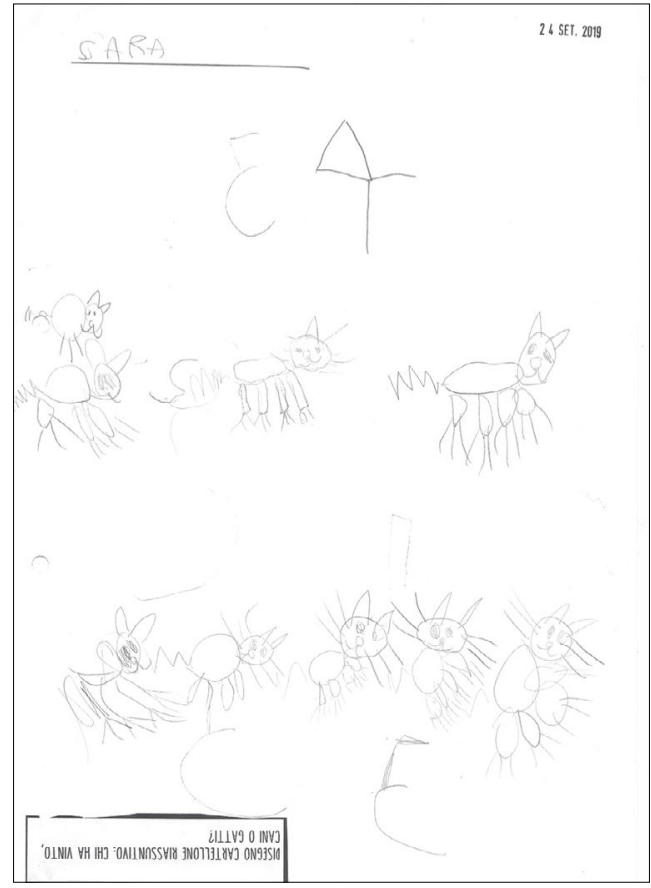

Figura 4. Sul proprio foglio ogni bambino rappresenta la sintesi dei dati raccolti.

\subsubsection{Indagine sul genere: ci sono più maschi o più femmine?}

In questa seconda parte relativa alla prima fase dell'itinerario, durata dalla prima settimana di ottobre alla metà di novembre 2019, la domanda che ha guidato le attività è stata: «Secondo voi ci sono più maschi o più femmine?». Da questo stimolo sono partite diverse attività.

I bambini hanno dapprima deciso di disporsi frontalmente su due file: da una parte i maschi e dall'altra le femmine. Questa strategia ha permesso di contare e valutare, anche solo visivamente, quale fosse il gruppo più numeroso. 
Più complesso è stato invece riflettere sulla maggioranza di genere rispetto all'intera sede, sia per l'elevato numero di bambini, sia considerato il fatto che durante il momento di lavoro i compagni più piccoli si trovavano a casa, mentre altri stavano svolgendo il riposo pomeridiano. I bambini hanno quindi deciso di lavorare a gruppi su grandi fogli divisi a metà e annotare i contrassegni di tutti gli allievi suddividendoli tra maschi e femmine (Figure 5 e 6 ).

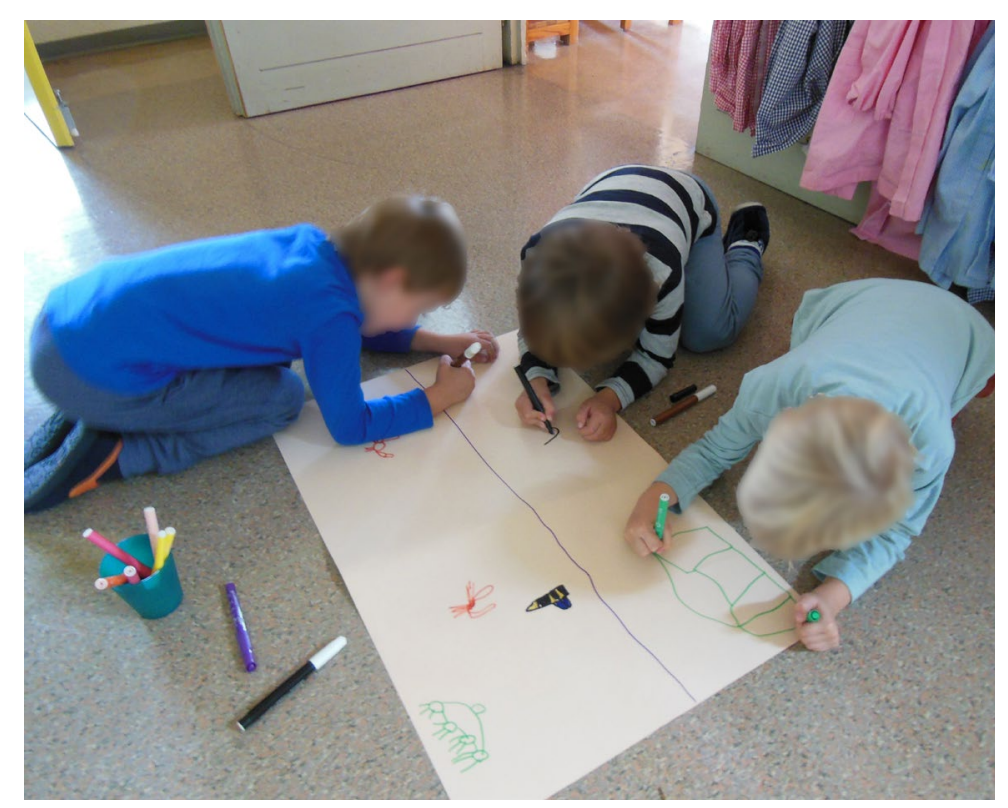

Figura 5. I bambini, divisi in gruppi, annotano i contrassegni di tutti gli allievi, suddividendoli per genere.

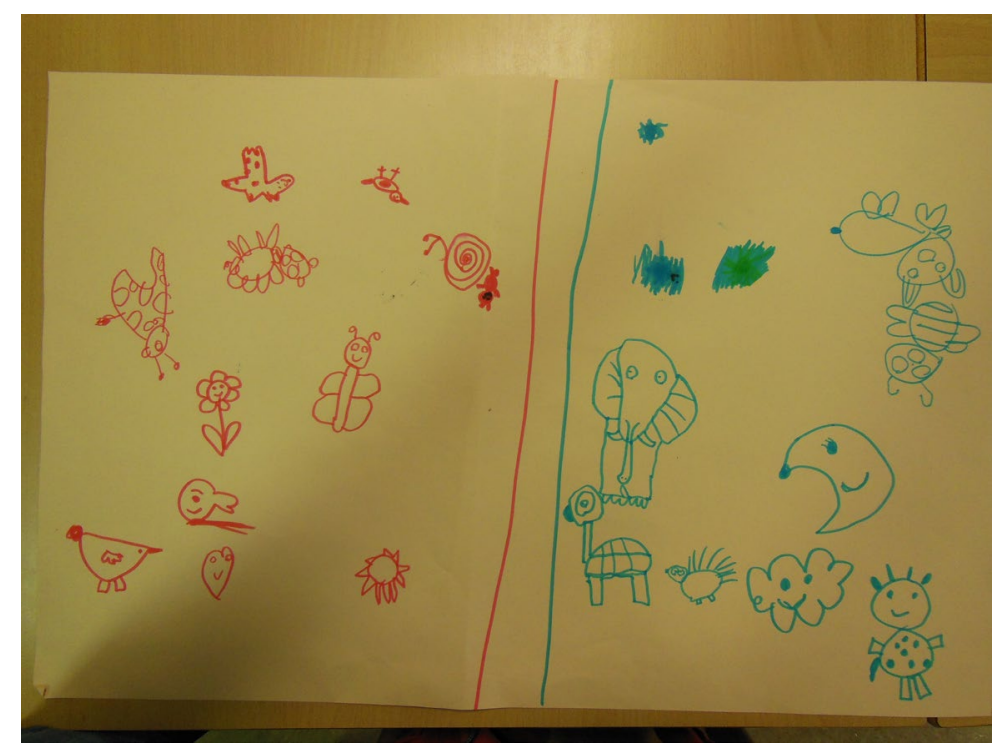

Figura 6. Contrassegni rappresentati su un foglio e suddivisi per genere.

Il problema è emerso in sede di sintesi dei dati: come unire e riportare questi dati su foglio o su un unico cartellone?

Per semplicità si è tornati per un momento a lavorare sul conteggio del gruppo dell'ultimo anno di scuola dell'infanzia, chiedendo ai bambini di trovare un modo per rappresentare il fatto che nel gruppo fossero presenti nove maschi e undici femmine (Figura 7). 


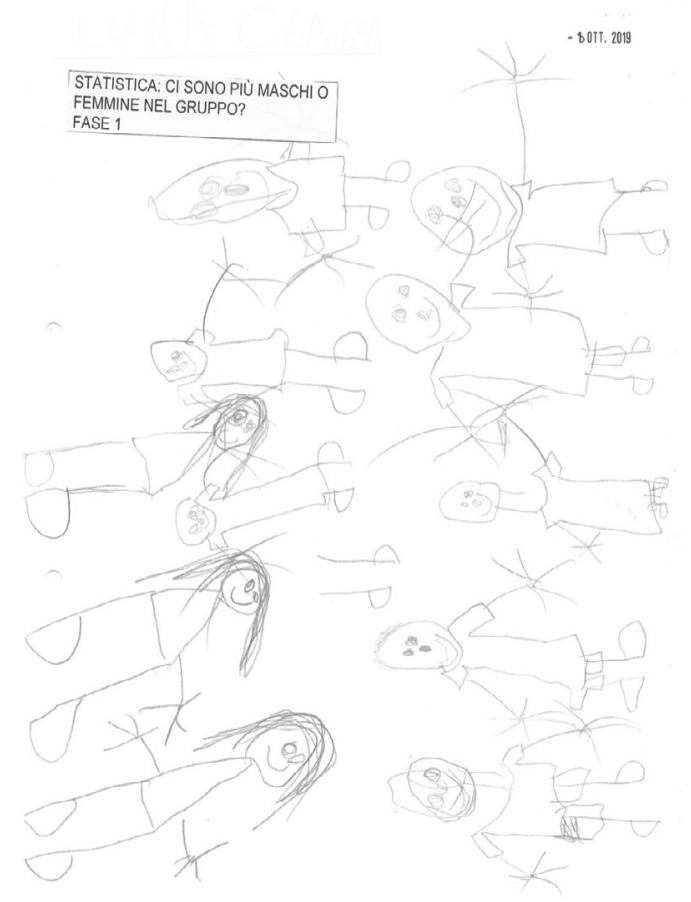

Figura 7. A coppie, i bambini rappresentano quanti maschi e quante femmine ci sono nel gruppo dell'ultimo anno di scuola dell'infanzia.

Dopo aver sperimentato il disegno dettagliato di ogni persona sul foglio, i bambini hanno capito che questa soluzione era troppo gravosa sia sul piano energetico, sia in termini di tempo: è nata I'esigenza di semplificare. II dato emerso conferma quindi quanto anticipato nel par. 1.2, a proposito della funzione comunicativa convenzionale del pittogramma, utile a facilitare il riconoscimento, le inferenze e la memorizzazione (Darras, 2005).

Da questa attività ha preso avvio una ricerca da parte dei bambini di figure umane stilizzate ed è venuta loro in aiuto l'immagine affissa sulle porte dei bagni, un esempio di pittogramma offerto dal contesto sociale della sezione scolastica. I bambini hanno copiato i due pittogrammi a mano libera e, in seguito, hanno scomposto i loro disegni in semplici figure geometriche del piano (Figura 8) per poi ricomporle con la tecnica del collage eseguita individualmente (Figure 9 e 10).

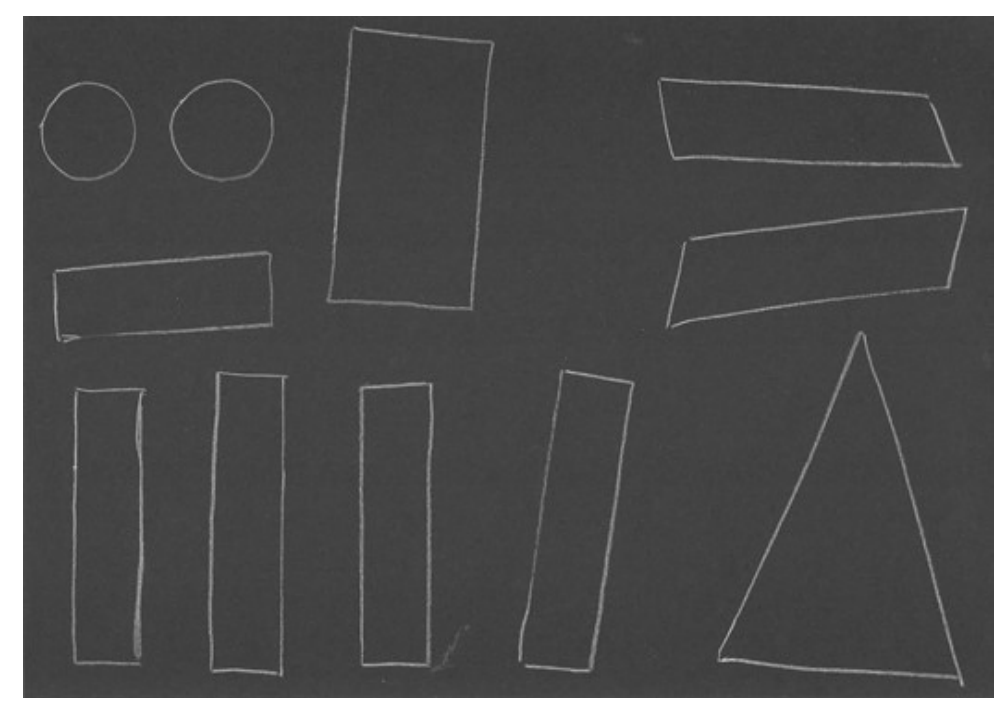

Figura 8. Figure geometriche nate dalla scomposizione delle figure umane stilizzate del bagno. 


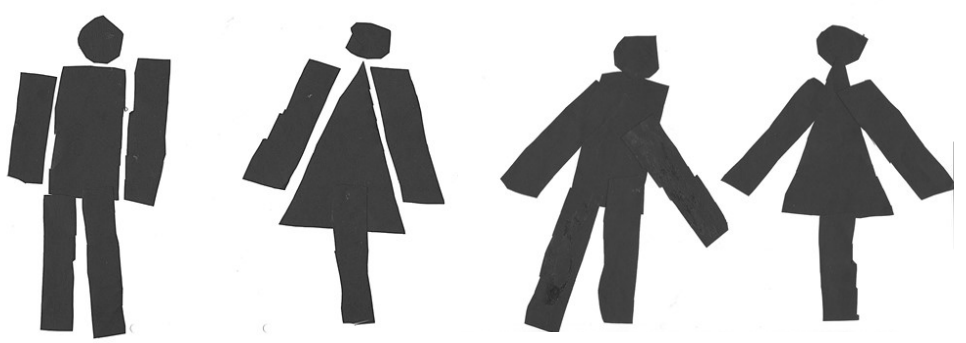

Figure 9-10. Collage di Federico e di Marta, ${ }^{1}$ che rappresentano le figure umane maschile e femminile realizzate ritagliando e assemblando le forme geometriche delle figure umane stilizzate del bagno.

A seguito di questo lavoro è stato più semplice trovare il modo di simbolizzare la figura umana e di conseguenza registrare i dati relativi ai maschi e le femmine. ${ }^{2}$

\subsection{Seconda fase: alla scoperta dei pittogrammi}

Questa fase dell'itinerario si è snodata lungo i primi due mesi del 2020. In continuità con le attività di simbolizzazione della figura umana sono stati introdotti i pittogrammi, in particolare quelli delle Ferrovie Federali Svizzere (https://digital.sbb.ch/de/brand elemente/piktogramme). II repertorio grafico-informativo delle FFS si iscrive nella tradizione visiva della cosiddetta Scuola svizzera di design, o Swiss Style, le cui figure preminenti possono essere individuate in Josef Müller-Brockmann (19141996), Armin Hofmann (1920-2020) e Karl Gerstner (1930-2017). Tale scelta è anche funzionale a un criterio di qualità nella proposta del referente didattico: come richiamato dal Piano di studio della scuola dell'obbligo ticinese (DECS, 2015), il contributo dei linguaggi artistici ed espressivi è da intendersi finalizzato anche alla «configurazione di adeguati riferimenti culturali» (p. 227).

I bambini, divisi in gruppi da 4, hanno visionato i pittogrammi delle FFS. || loro compito era di commentarli, trovare un modo per categorizzarli (ad esempio per tipologia: mezzi di trasporto, oggetti, animali ecc.) e, successivamente, presentarli ai compagni. Divisi in questi piccoli gruppi, i bambini hanno preso visione, commentato e suddiviso in categorie i pittogrammi ricevuti. In seguito hanno provato a riprodurli con il vincolo di utilizzare unicamente le forme geometriche date, che avrebbero dovuto ritagliare e incollare su cartoncini blu. Unica eccezione: delle semplici linee tracciate con la matita.

In un secondo momento ogni bambino ha scelto uno dei pittogrammi e ha provato a riprodurlo utilizzando le figure geometriche già presenti nella fase precedente (simbolizzazione della figura umana). La difficoltà maggiore è stata adattare delle forme standard alle figure e il vincolo di limitare gli interventi su di esse con la matita o altri strumenti grafici (Figura 11).

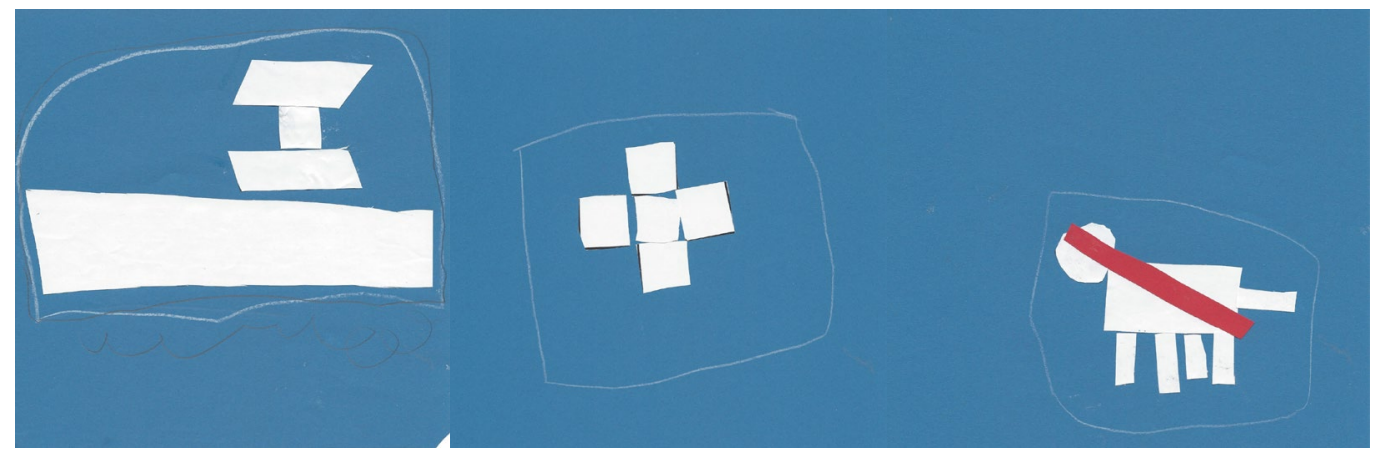

Figura 11. Esempi di pittogrammi riprodotti dai bambini partendo da forme geometriche.

1. Per la tutela della privacy degli studenti coinvolti, i nomi sono stati cambiati.

2. Questa attività è andata a toccare temi e stereotipi legati all'identità di genere. In questo senso, nelle conclusioni si approfondisce un dato emerso grazie all'attività di collage realizzata dai bambini. 
Esaurito il discorso sui pittogrammi delle FFS si è presentata l'opportunità dell'imminente inaugurazione della sede ristrutturata della scuola elementare per lavorare sull'invenzione di pittogrammi in relazione ad aule e spazi. Il compito di creare dei pittogrammi è stato proposto ai bambini e alle bambine come una sfida per identificare i diversi ambienti del nuovo edificio scolastico. Dopo la visita alla sede ristrutturata, i bambini sono stati invitati a elencare i locali visionati. Da questa lista a coppie i bambini hanno scelto uno spazio e provato dapprima a progettare una forma iconica che lo rappresentasse e in seguito a realizzarla, sempre attraverso l'utilizzo delle forme geometriche (Figure 12 e 13).
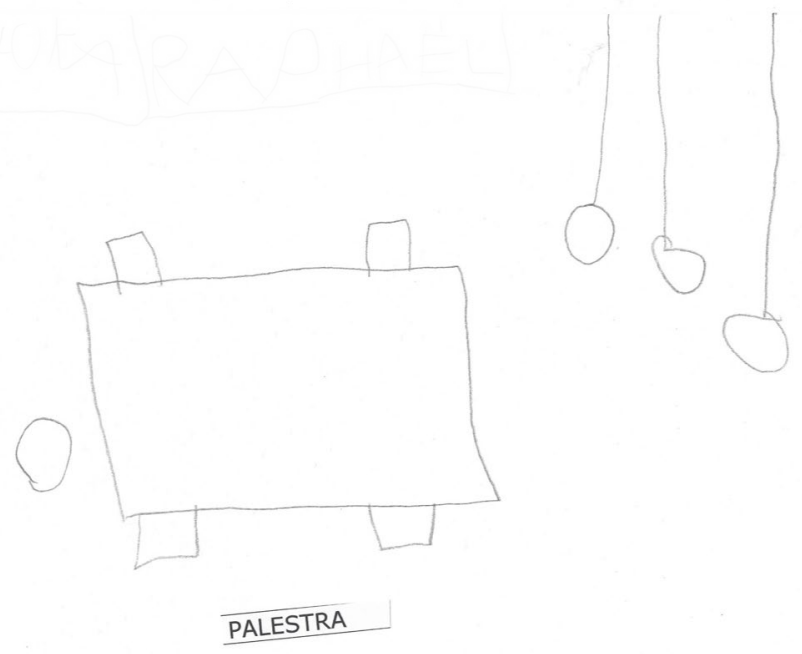

Figura 12. Progetto di un pittogramma che rappresenta la palestra.

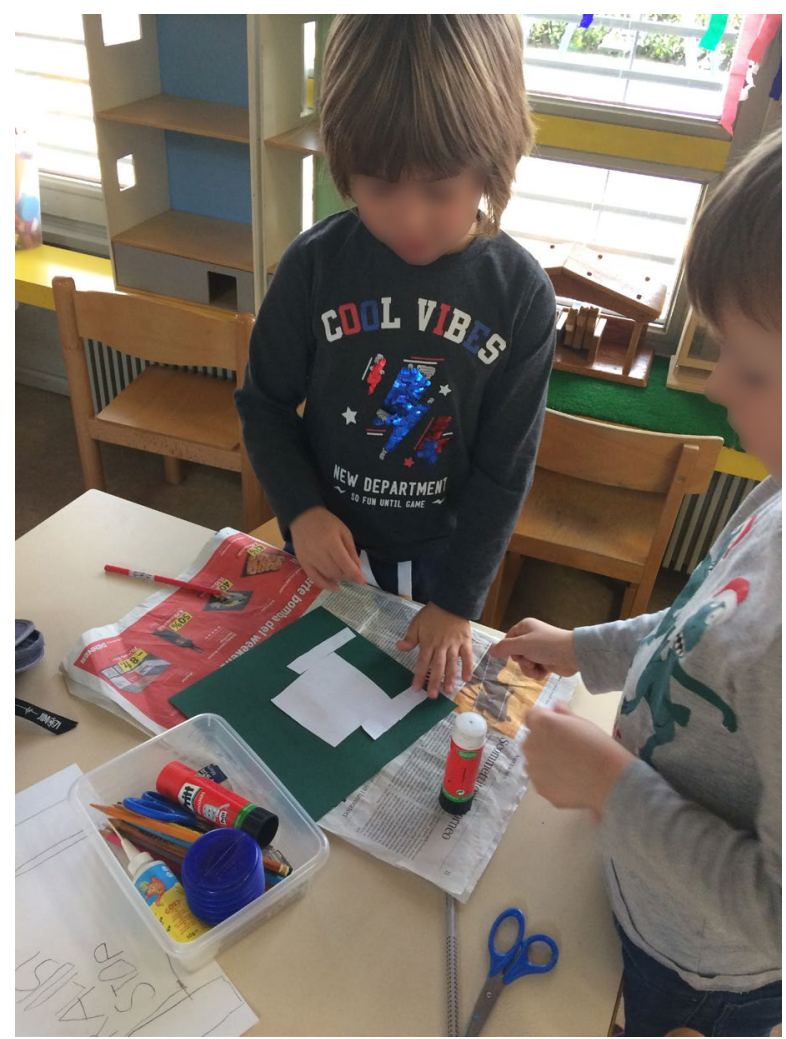

Figura 13. A coppie, i bambini realizzano, attraverso l'uso di forme geometriche, il pittogramma di un'aula o di un altro spazio scolastico. 


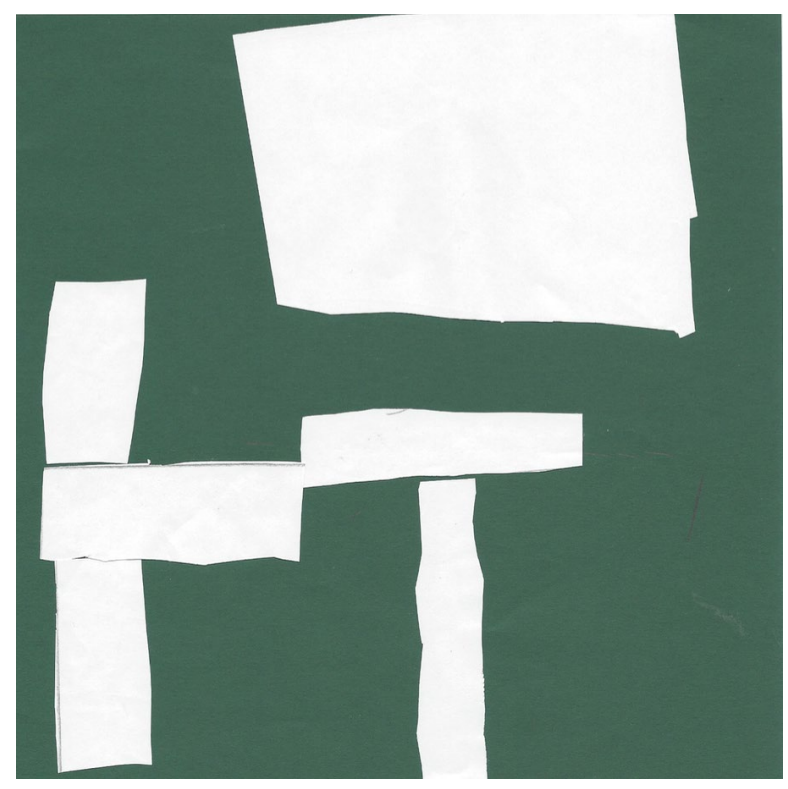

Figura 14. Esempio del pittogramma che rappresenta l'aula di classe.

Il nuovo tipo di immagine richiesta dal compito (rappresentazione spaziale di ambienti) ha fatto emergere ostacoli supplementari legati alla stilizzazione, semplificazione e schematizzazione della realtà (Figura 14). Si è reso necessario un lavoro, tramite discussione, per cogliere le parti essenziali e caratterizzanti di ogni spazio: ad esempio il flauto e le note per l'aula di educazione musicale, un banco e una sedia per l'aula di classe.

I lavori finiti avrebbero dovuto essere appesi nei vari ambienti in occasione dell'inaugurazione (Figura 15), che purtroppo non ha potuto avere luogo a causa dell'emergenza sanitaria.
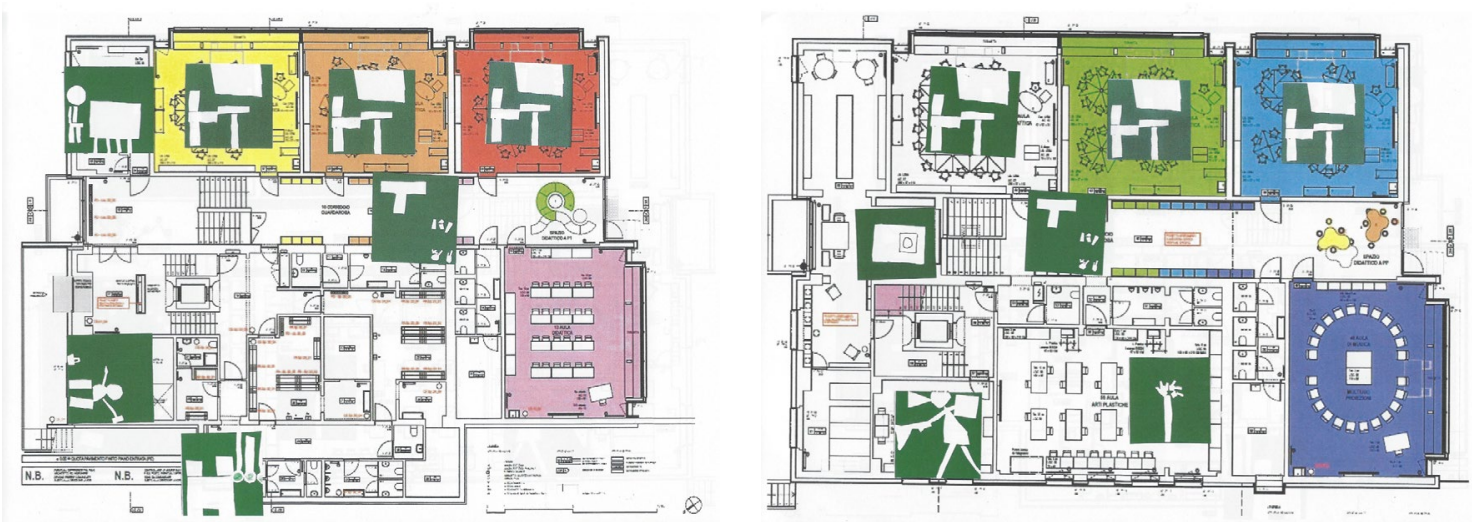

Figura 15. Planimetria della scuola elementare sulla quale sono stati applicati i pittogrammi relativi agli spazi creati dai bambini.

\subsection{Terza e ultima fase: creiamo disegni con le sagome}

Al rientro dal lockdown, tra metà maggio e metà giugno 2020, i bambini della sezione hanno fatto ritorno in presenza a effettivi ridotti, metà alla volta, a causa della delicata situazione legata alla pandemia. Tutti si sono dedicati ad un nuovo lavoro di rappresentazione visiva basato su sagome, o mascherine, realizzate su misura. Come illustrato nella Figura 16, le mascherine permettevano di creare forme più o meno convenzionali come la goccia, l'ogiva, l'uncino, il triangolo, il cerchio e il rombo; ognuna di quattro grandezze diverse. 


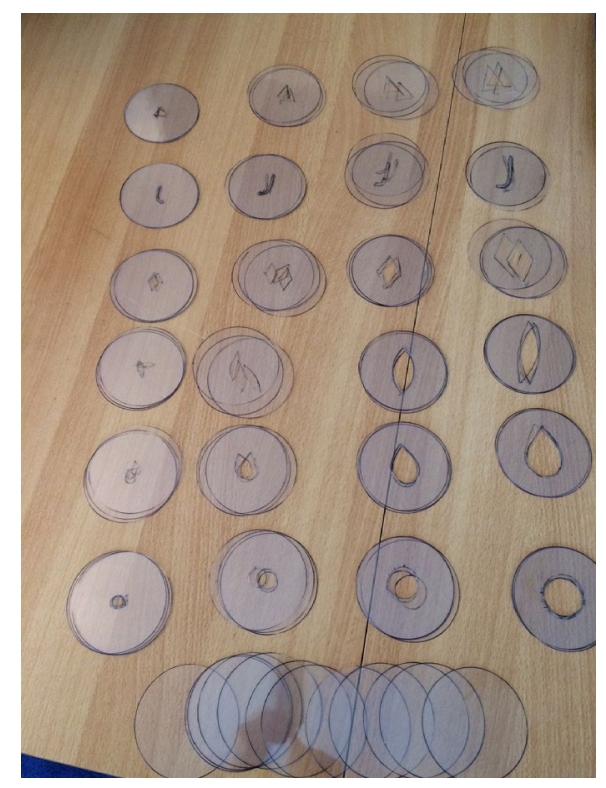

Figura 16. Sagome di plastica semirigida create su misura.

Per gli allievi, la motivazione alla base dell'attività era quella di creare delle figure di uccelli, tema dell'anno scolastico, che dovevano poi essere proposte ai compagni per un riconoscimento sotto forma di indovinello.

Nel lavoro di stilizzazione è stato fondamentale estrapolare le caratteristiche essenziali e caratterizzanti di ogni animale, per poi cercare la forma che più si addiceva alla rappresentazione grafica di una data parte del corpo.

In prima battuta c'è stato un momento di scoperta delle sagome e di sperimentazione libera (Figura 17), con relativa discussione sul loro uso e sulle strategie emerse. Dalle Figure 18 e 19 si nota come alcuni codici del linguaggio visivo (simmetria, ritmo e modularità), mobilitati spontaneamente dai bambini giustapponendo e sovrapponendo le forme per creare diversi motivi, contribuiscono alla creazione di artefatti ancora indeterminati fra la funzione decorativa e quella figurativa dell'immagine.

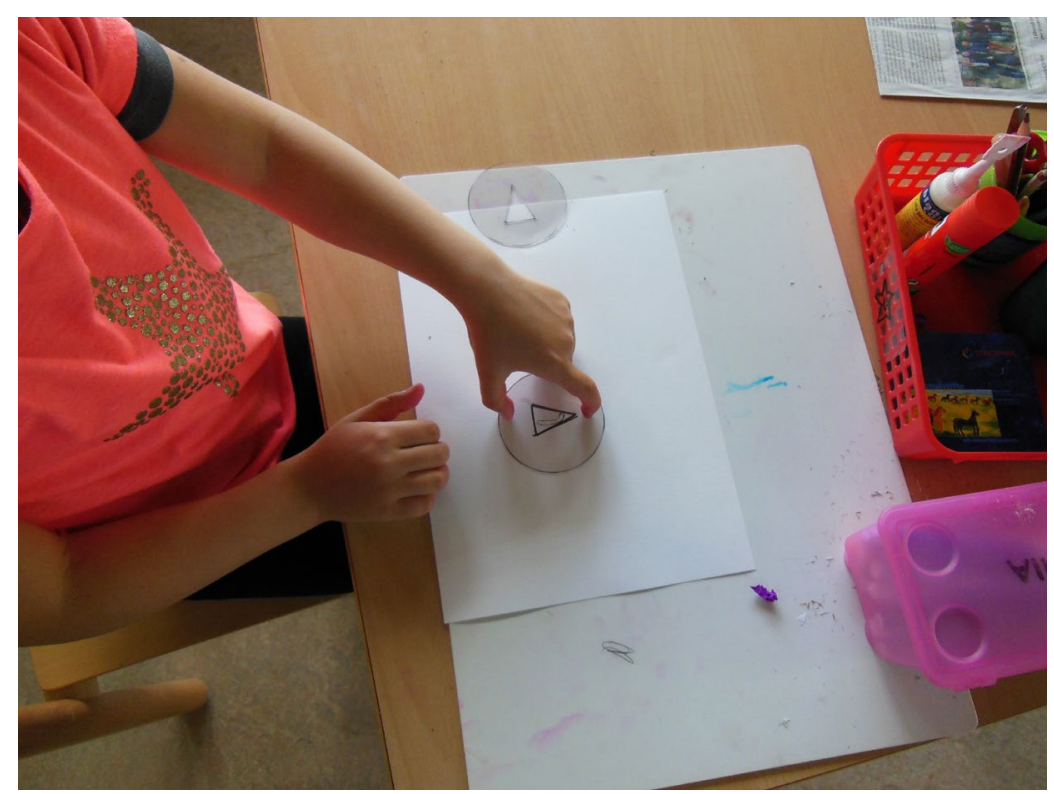

Figura 17. I bambini sperimentano liberamente con le sagome. 

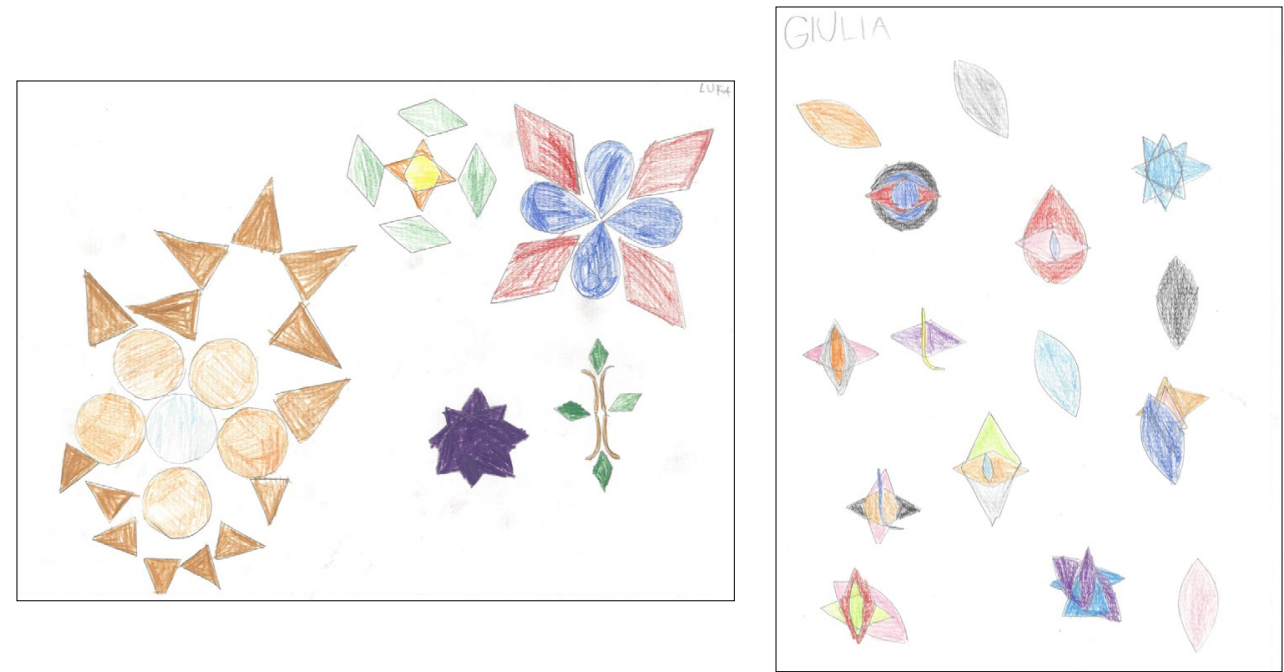

Figure 18-19. Due prodotti della sperimentazione libera.

Nell'attività seguente ogni bambino ha ricevuto un uccello diverso da riprodurre, orientando così in modo esplicito l'intenzione della rappresentazione grafica in chiave figurativa (Figure 20 e 21).

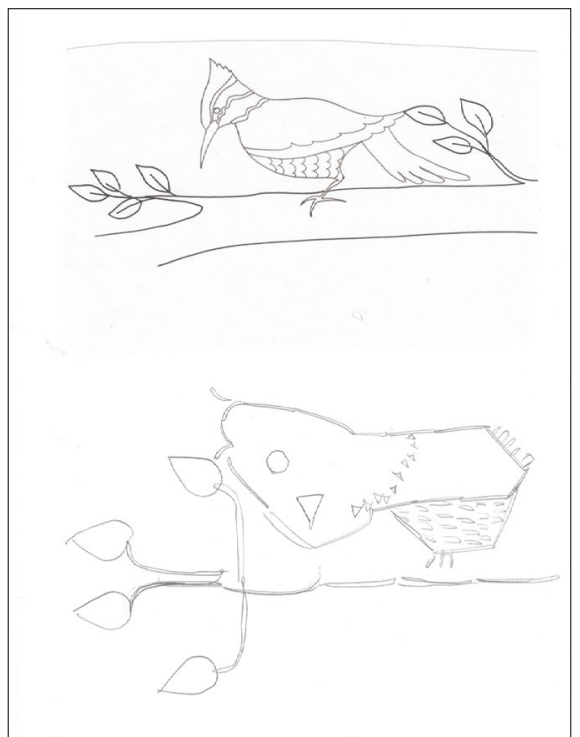

Figura 20. II picchio rappresentato attraverso I'utilizzo delle sagome.

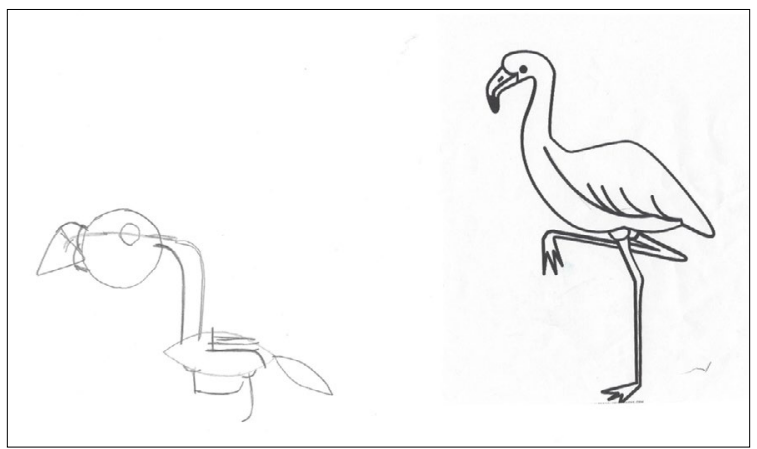

Figura 21. II fenicottero rappresentato attraverso l'utilizzo delle sagome. 
Parallelamente sono stati svolti degli allenamenti rappresentando, sempre attraverso I'uso delle mascherine, le varie parti del corpo che caratterizzano un uccello: piumaggio, ali, becco, zampe, occhi (Figura 22).

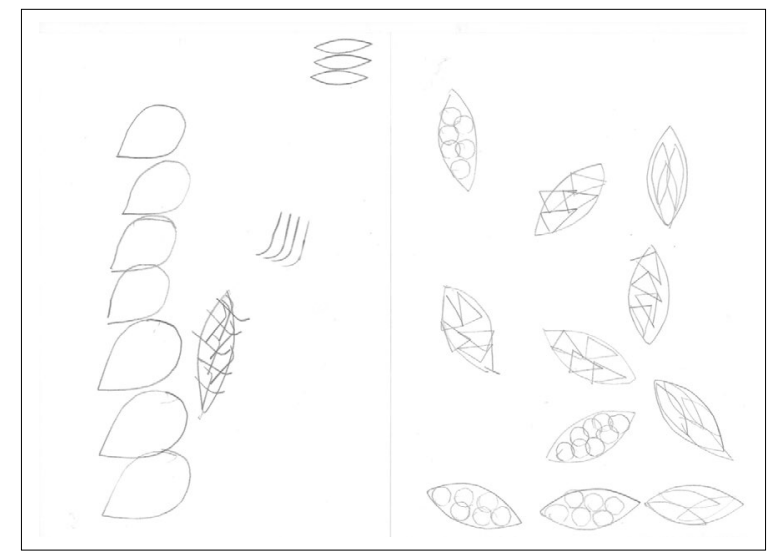

Figura 22. Esempio di rappresentazione per le ali attraverso l'utilizzo delle sagome.

Nel corso della sperimentazione con le sagome, finalizzata alla creazione di figure di uccelli, le strategie messe in atto dalle allieve e dagli allievi sono state principalmente quelle di accostare, giustapporre e combinare le forme per crearne di nuove e più somiglianti all'oggetto di studio, sia nel caso della figura intera (Figure 20 e 21), sia di una parte di esso (Figura 22). In alcuni casi i bambini hanno utilizzato solo una parte della mascherina (Figura 20: alcune linee rette sono state tracciate con una parte della forma a uncino).

\section{Conclusioni}

In generale si è rilevato un marcato interesse nel gruppo di allievi lungo tutto il percorso. I bambini sono apparsi fin da subito coinvolti e motivati nelle varie attività. Hanno avuto spesso l'occasione di lavorare in piccoli gruppi o a coppie, raramente a livello individuale, e questo ha sicuramente favorito il rafforzamento delle relazioni interpersonali, la collaborazione e l'autonomia delle allieve e degli allievi. Gli apprendimenti principali che le tre parti dell'itinerario hanno stimolato sono legati all'astrazione, alla capacità di rilevare ed estrapolare l'essenziale e a quella di simbolizzare e sintetizzare.

\subsection{Apprendimenti legati alla matematica}

L'itinerario proposto ha permesso ai bambini e alle bambine, grazie alla prima attività, di svolgere in prima persona un processo statistico, dalla domanda di ricerca fino alla rappresentazione dei risultati, combinando, in un'unica attività, aspetti di classificazione (compresa la rappresentazione di classi di equivalenza), conteggio e rappresentazione numerica, favorendo una creazione di senso dei diversi concetti mobilitati. L'attività ha pure messo in luce come, in questo caso, le difficoltà principali riscontrate nella risoluzione delle situazioni problema in matematica non siano legate necessariamente ad aspetti matematici, quanto piuttosto ad aspetti relativi alla rappresentazione e alla comunicazione. Questa considerazione è un'ulteriore evidenza a favore della concezione e realizzazione di itinerari interdisciplinari. Le attività successive hanno invece permesso agli allievi e alle allieve di aumentare la 
familiarità con I'utilizzo di diverse figure geometriche, sia standard che non standard, favorendo così anche in questi casi la creazione di senso e l'astrazione.

\subsection{Apprendimenti legati all'educazione visiva}

In linea con gli autori cui si è fatto riferimento nel par. 1.2, si conferma l'utilità didattica di integrare, nel lavoro per la definizione dei concetti, i modi della significazione grafica con quelli di altri linguaggi come parole e numeri. La permeabilità e l'interscambio fra i processi della percezione visiva e della categorizzazione astratta dei concetti è un dato che si è potuto notare chiaramente. È poi emerso un dato specifico interessante, come anticipato nel par. 3.1.2, che riguarda la distinzione di genere richiesta ai bambini nella fase di simbolizzazione della figura umana mediante la tecnica del collage. La maggioranza delle configurazioni femminili (19 su 20) è stata schematizzata con un unico elemento rettangolare equivalente alle gambe, laddove la configurazione maschile presenta due rettangoli paralleli, a volte divaricati oppure leggermente distanziati (Figure 9 e 10). La motivazione sembra banale: avendo a disposizione solo 7 elementi rettangolari (Figura 8) adatti alla resa visiva di braccia e gambe - quando ne sarebbero serviti 8 - i bambini sono stati obbligati a scegliere quale delle due figure, donna o uomo, rappresentare a gambe unite. Curiosamente, però, nessun bambino ha scelto di schematizzare l'uomo con le gambe unite; dai disegni spontanei, per contro, emergeva una maggiore uniformità degli iconotipi di genere, simbolicamente rappresentati entrambi sempre con due gambe, indifferentemente se uomo o donna (Figura 7). Ora, lo stesso fenomeno è stato studiato da Darras, che commenta:

\footnotetext{
«La rappresentazione delle gambe degli uomini lascia supporre che indossino dei pantaloni. Al contrario, la rappresentazione delle gambe delle donne è spesso più serrata, il che, associato al fatto di portare un vestito o una gonna, fa supporre che le gambe siano nude. Le gambe sono generalmente disegnate parallele, ma possono convergere in alcuni pittogrammi femminili. Sono pudicamente tenute insieme nei pittogrammi femminili, mentre possono essere ostentatamente aperte in quelli maschili».
}

(Darras, 2005, p. 2, traduzione degli autori)

Possiamo ipotizzare che la scelta di rappresentazione dei bambini sia la conseguenza di uno schema appreso - la segnaletica dei bagni della scuola - e non spontaneo, come dimostrato in effetti dai disegni iniziali. In chiave di pensiero critico e riflessivo, quindi, s'impone una riflessione sugli stereotipi di genere, mentre si richiama la necessità per l'adultità educativa di riflettere sulle proprie conoscenze implicite, specie quando siano veicolate da strutture visive oltre che verbali. Darras (2005) prospetta I'opportunità, nello specifico dei pittogrammi di genere, di approfondire l'analisi didattica dei materiali implementando un approccio semiotico centrato sulla relazione sociale e culturale (contesto teorico, sociale e storico): "questi segni di relazione risultano da scelte e selezioni operate a tutti i livelli di produzione e di interpretazione. Sono dettati o guidati dallo stato delle conoscenze e delle relazioni di cooperazione, negoziazione e potere nella società» (Darras, p. 9, traduzione degli autori). Per quanto innocui possano sembrare, i pittogrammi realizzati dai bambini nei contesti di realtà che hanno caratterizzato il percorso qui presentato, svolgono la duplice funzione di «registratori e prescrittori della tradizione» (Darras, p. 9, traduzione degli autori).

In generale, l'adozione di un approccio contestuale nella progettazione di questo itinerario ha consentito di realizzare attività interdisciplinari che hanno permesso alle allieve e agli allievi di acquisire diversi apprendimenti, come testimoniato dalle diverse immagini riportate nel presente articolo. D'altro canto, tale approccio ha richiesto di poter gestire in modo dinamico le competenze mirate, adattandole, rivedendole e addirittura concependole man mano che gli allievi e le allieve procedevano nel 
loro percorso di apprendimento. Raccomandiamo dunque di adottare un approccio contestuale solo se non si è troppo vincolati a delle precise competenze mirate da consolidare lungo tutto l'itinerario e se si dispone di ampia autonomia nella concezione e realizzazione degli itinerari didattici.

\section{Bibliografia}

Darras, B. (1998). L'image, une vue de l'esprit. Étude comparée de la pensée figurative et de la pensée visuelle. Recherches en communication, 9, 77-99.

Darras, B. (2005). Semiotic of visual signs and information design. Information Design International Conference Proceedings. https://www.academia.edu/27840113/Darras B 2005 Semiotic of visual signs and information design

Dipartimento dell'educazione, della cultura e dello sport. (2015). Piano di studio della scuola dell'obbligo ticinese. Divisione scuola, DECS.

Klassen, S. (2006). A theoretical framework for contextual science teaching. Interchange, 37(1-2), 31-62.

Massironi, M. (1982). Vedere con il disegno. Aspetti tecnici, cognitivi, comunicativi. Franco Muzzio.

Meisser, S. (2010a). Perché i cani odiano i gatti. Fatatrac.

Meisser, S. (2010b). Perché i gatti odiano i cani. Fatatrac.

Piatti, A. (2015). II senso e il significato dei concetti in matematica. Bollettino dei docenti di matematica, 70, 103-106.

Radford, L., Schubring, G., \& Seeger, F. (2011). Signifying and meaning-making in mathematical thinking, teaching and learning. Educational Studies in Mathematics, 77, 149-156.

Rickenmann, R. (2001). Sémiotique de l'action éducative: apports pour l'analyse didactique des leçons d'arts plastiques. In J. M. Baudouin (Eds.), Théories de I'action et éducation (pp. 225-253). De Boeck Supérieur. https://doi.org/10.3917/dbu.baudo.2001.01.0225 